gonorrhoea and Chlamydia screening. Prevalence of condyloma acuminatum, HIV, gonorrhoea and Chlamydia were performed. Analysis unites and multivariates in logistic regression have been performed to estimate the influence of these factors in the appearance of condyloma acuminatum among FSWs in DIST.

Results Among the 403 FSWs enrolled, the prevalence of condyloma acuminatum was $5.2 \%$. The one of HIV infection was $37 \%$. Prevalence of gonorrhoea and Chlamydia were nil. This remark certifies the high level of condom use (87.3\%) among FSWs. HIV infection was significantly associated with appearance of condyloma acuminatum in the cohort of FSWs attending the STI dispensary with an adjusted OR of 2.4 ( $95 \% \mathrm{CI}=0.90$ to 6.43$)$.

Conclusion Condyloma acuminatum remains a way in HIV infection transmission. A meticulous check and treatment contributes to reduce HIV/AIDS risk.

\section{P1-S2.16 WHAT DO FEMALE SEX WORKERS DO IF THEY HAVE GENITAL SYMPTOMS?}

doi:10.1136/sextrans-2011-050108.73

${ }^{1} \mathrm{~K}$ Lee, ${ }^{2} \mathrm{~K}$ H Wong. ${ }^{1}$ Stanley Ho Centre for Emerging Infectious Diseases, Hong Kong; ${ }^{2}$ Special Preventive Programme, Hong Kong

Background Female sex workers (FSW) is an important population in sexually transmitted infection (STI) epidemiology because of their extended partnership pattern that possibly leads to rapid STI spread. Prompt STI diagnosis, treatment and surveillance among FSW is therefore necessary for effective control of STI in the community, and public STI clinics remain the key platform for such. Yet, much is unknown about the pattern of health-seeking behaviour including STI clinic attendance of the FSW.

Methods A cross-sectional survey of 986 FSWs sampled from onewoman brothels, bars, clubs and streets in Hong Kong were conducted in 2009. Questions on last year attendance at public STI clinics, NGO testing centres, prior 6-month self-reported STI, STI symptoms and the subsequent actions regarding the symptoms were asked. Descriptive analysis was performed to show these frequencies. Self-reported rate among those with or withot symptoms were calculated. Self-reported STI and STI symptoms among those STI clinic and NGO attendees and non-attendees were compared using $\chi^{2}$ test.

Results Overall, $7.8 \%$ respondents reported having at least one episode of STI in the last 6 month. About one-third reported either having abnormal vaginal discharge, growth or ulcer in recent 6 months. Strikingly, about two-thirds would either self-medicate or adopt wait and see approach and about one-third attended private doctor or doctor across the border. Altogether less than 5\% would attend NGO/STI clinic. Only 26\% reported attendance at public STI clinic and $25 \%$ in NGO testing centre in the past 1 year. Selfreported STI was reported by $4 \%$ of those asymptomatic respondents, and 14\% among those reported having any genital symptoms $(p<0.05)$. The self-reported STI rate and STI symptom pattern did not differ significantly between the public STI and NGO attendees and the non-attendees see Abstract P1-S2.16 Figure 1.

Conclusions STI are three times more commonly reported among FSWs who reported genital complains. However, majority of them would not seek appropriate medical care when symptoms occur indicating that treatment might have been delayed. Self-medication is common and remain a challenge for health promotion in FSW. Although no significant difference is found between clinic attendees and non-attendees on STI symptoms, STI clinic data only captures a fraction of STI burden in FSW.
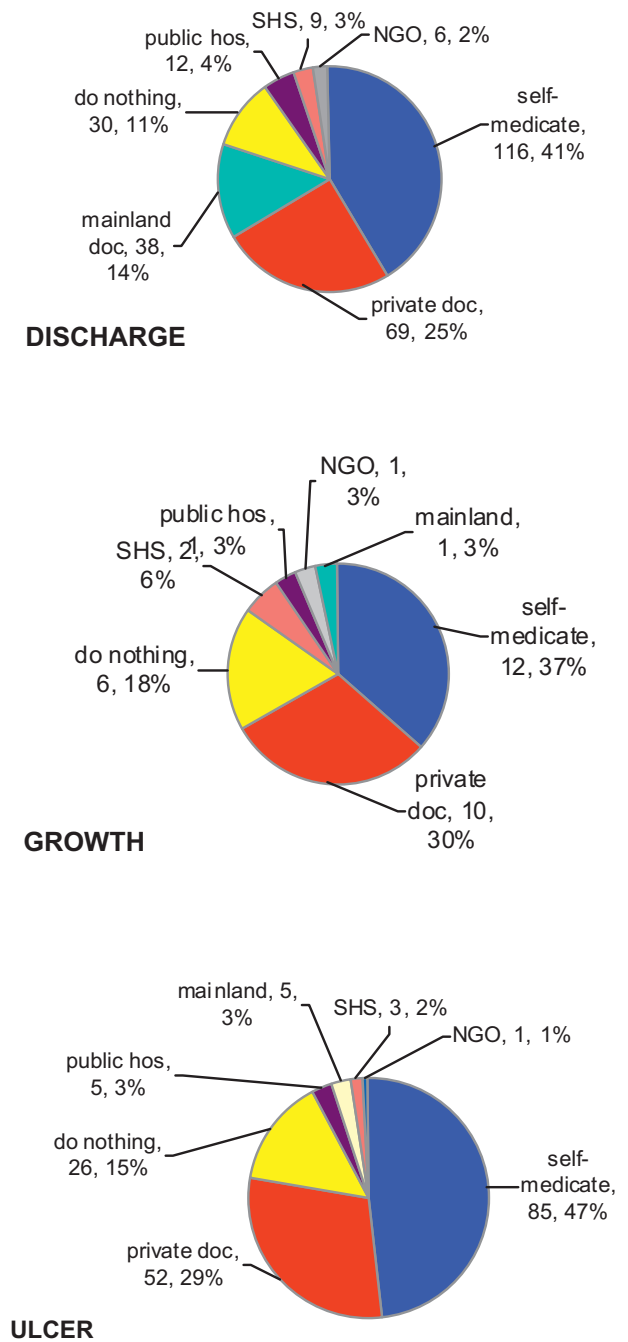

Abstract P1-S2.16 Figure 1 Actions subsequent to presence of genital symptoms (vaginal discharge, genital growth or ulcer). SHS, public STI clinic.

\section{P1-S2.17 MODELLING THE IMPACT OF RAPID TEST SYPHILIS SCREENING AMONG FEMALE SEX WORKERS IN CHINA}

doi:10.1136/sextrans-2011-050108.74

Kate Mitchell, Andrew Cox. LSHTM, London, UK

Background In China, female sex workers (FSWs) are hard to reach for public health interventions. STIs and syphilis (Tp) in particular, are now a major public health concern in China. Currently, diagnosis of active $\mathrm{Tp}$ requires laboratory tests, which have high sensitivity, are capable of diagnosing active Tp infection, but involve delays between testing and treatment. Such delays lead to a low proportion of FSWs returning for treatment. In contrast, rapid Tp tests can provide instant results, thereby offering the possibility of immediate treatment, but are generally lower sensitivity and cannot differentiate between active and previous Tp infection. In this study, we estimate the impact of using rapid tests to screen FSWs for Tp in a Chinese setting and compare the performance with lab-based diagnosis.

Methods A deterministic model of Tp transmission among FSWs and clients was parameterised and fitted to data from the medium prevalence setting (Kaiyuan City). Random sampling of parameter uncertainty ranges was used to find multiple fits. Model fits were used to explore the epidemiological impact of providing rapid $\mathrm{Tp}$ 